\title{
Author Correction: Synthesizing ginsenoside Rh2 in Saccharomyces cerevisiae cell factory at high- efficiency
}

Pingping Wang, Wei Wei, Wei Ye, Xiaodong Li, Wenfang Zhao, Chengshuai Yang, Chaojing Li, Xing Yan and Zhihua Zhou

Correction to: Cell Discovery (2019) 5:5

https://doi.org/10.1038/s41421-018-0075-5

Published online 15 January 2019

Following publication of the original article ${ }^{1}$, an error was identified in the "Acknowledgements" section. The funding number for "the National Mega-project for Innovative Drugs (2017ZX09101003-006-002)" is wrong; the correct one is the National Mega-project for
Innovative Drugs (2018ZX09711001-006-002). We are sorry for the mistake.

Published online: 07 December 2020

\section{Reference}

1. Wang, P. et al. Synthesizing ginsenoside Rh2 in Saccharomyces cerevisiae cell factory at high-efficiency. Cell Discov. 5, 5 (2019). 